\title{
SUPERHUB: Integrating Digital Behaviour Management into a Novel Sustainable Urban Mobility System
}

Simon Wells, Paula Forbes, Judith Masthoff

Department of Computer Science

University of Aberdeen

Aberdeen, UK

firstname.lastname@abdn.ac.uk

\author{
Silvia Gabrielli \\ CREATE-NET \\ Via Alla Cascata, 56/D \\ Trento \\ Italy \\ silvia.gabrielli@create-net.org
}

\author{
Antti Jyllha \\ Department of Computer Science \\ University of Helsinki, P.O. Box 68 \\ FI-00014 University of Helsinki \\ Finland \\ Antti.jylha@helsinki.fi
}

In this paper we present SUPERHUB, a prototype, open platform for urban-mobility that integrates multimodal journey planning with captology influenced digital behaviour management in order to encourage increasingly sustainable travel behaviours. We build upon recent research in digital persuasion, mobile $\mathrm{HCl}$, and findings from a user-centered design process to create a novel and sustainable urban-mobility platform that comprises an integrated server platform with an open API, Android mobile client, and a responsive web interface. Our goal is to support our users in realising their expressed sustainable travel goals.

captology, sustainability, urban transport, digital behaviour management

\section{INTRODUCTION AND MOTIVATION}

Effective and efficient travel within an urban environment is an increasingly complex undertaking. Whilst there are many varied digital tools that support urban travellers, discovering these tools and using them effectively is a complex and time consuming task for the end user. With increasingly urban populations and with transportation, both freight and human mass-transit together, accounting for $30 \%$ of EU energy consumption and taking into account the resultant greenhouse gas emissions, it is clear that more efficient and sustainable urban mobility will have real, tangible benefits for urban populations.

To this end, the SUPERHUB ${ }^{1}$ project is constructing an open platform that integrates core mobility tools, such as journey planning, with behaviour management tools that support travellers who want to improve the sustainability of their transport choices [Forbes et al. (2012)]. Because urban travellers usually have a range of mobility options available to them, including a range of personal and mass transportation options, journey planning must be aware of multiple transport modes [Hrncir and Jakob (2013)]. The most efficient and least environmentally impacting plans may cover a range of modes and where there are a range of potential plans, the system should support the user by matching potential plans to the user's expressed preferences and presenting a tractable number of plans for comparison, essentially recommending plans that are personalised to an individual SUPERHUB user. Additionally, a user's expressed travel preferences may be in conflict with their environmental preferences; the user wants to reduce their environmental impact, but the comfort and convenience of driving their own car is more important. This is where SUPERHUB aims to use captology [Fogg (2003)] based behaviour change management to help our users to effect lasting behaviour change [Gabrielli et al. (2013b)] using a range of behaviour change strategies [Gabrielli et al. (2013a)] as it has been shown that using a specific behaviour change theory can lead to more effective interventions [Webb (2010)].

In the SUPERHUB project we propose that travel tools should work well together, through a mixture of solid core platform integration and effective blending of external, third-party data sources. Blending disparate functionality and presenting data to users in a way that is both usable and that users want to

${ }^{1}$ http: //superhub-project.eu 
use is a core human computer interaction and user experience issue.

\section{THE SUPERHUB PLATFORM}

The SUPERHUB platform currently incorporates the following components:

- A multi-modal journey planner that enables users to search for journey plans that use a range of transportation modes.

- A recommendation engine that prioritises the ordering of generated plans according to the users' explicit mobility preferences.

- A persuasion engine for digital behaviour management that uses explicit goal setting and behaviour tracking to help users to increase the sustainability of their travel behaviours.

- An open Application Programming Interface (API) that is used both by official SUPERHUB mobile clients to interact with SUPERHUB data and functionality but also is the primary means by which the platform is open for external integration.

- An official Android mobile client that uses the API and exposes SUPERHUB functionality to end-users.

- A responsive web interface aimed at all users so that they can interact with the system using alternatives to the mobile client.

These core features have also been supplemented with ancillary functionality that enhances the enduser experience such as disruptive event reporting and reviewing. This provides support for a blend of practical, planned travel behaviours, with the flexibility to respond to changing traffic network conditions.

\section{DESCRIPTION OF THE DEMONSTRATION}

The demonstration comprises the following: (1) A virtual machine based demonstration server running selected components from the SUPERHUB backend, (2) An Android handset running the SUPERHUB Android mobile client, and (3) A laptop running the responsive web interface.

Because the full platform requires live data from external public transport network databases as well as large datasets to support mapping and geocoding, the demonstration server is configured to run off-line for reasons of reliability and practicality. If network access at the demonstration venue is sufficient then demonstrations from a live SUPERHUB deployment can be made. Our demonstration will provide an opportunity for delegates to utilise some of the SUPERHUB functionality, to explore firsthand the issues associated with integrating disparate technically adventurous computational functionality with usable mobile designs, and to discuss with us the design decisions that we have made so far.

\section{ACKNOWLEDGEMENTS}

This research is funded by the European Union under the Seventh Framework Programme (FP7) and is an output of the SUPERHUB Integrated Platform Project (289067). The full list of consortium members is available from http://www.superhub-project. eu.

\section{REFERENCES}

B. J. Fogg. Persuasive Technology: Using Computers to Change What We Think and Do (Interactive Technologies). Morgan Kaufmann Publishers, 2003.

P. J. Forbes, S. Wells, J. Masthoff, and H. Nguyen. Superhub: Integrating behaviour change theories into a sustainable urban-mobility platform. In Using Technology to Facilitate Behaviour Change and Support Healthy, Sustainable Living at BCS HCl 2012, 2012.

S. Gabrielli, R. Maimone, P. Forbes, J. Masthoff, S. Wells, L. Primerano, G. Bo, M. Pompa, and L. Haverinen. Co-designing motivational features for sustainable urban mobility. In ACM SIG$\mathrm{CHI}$ Conference on Human Factors in Computing Systems (CHI 2013), 2013a.

S. Gabrielli, R. Maimone, P. Forbes, and S. Wells. Exploring change strategies for sustainable urban mobility. In Designing Social Media for Change at the ACM SIG-CHI Conference on Human Factors in Computing Systems (CHI 2013), 2013b.

J. Hrncir and M. Jakob. Generalised TimeDependent Graphs for Fully Multimodal Journey Planning (to appear). In Proceedings of IEEE Intelligent Transportation Systems Conference (ITSC), 2013.

T. L. Webb. Using the internet to promote health behaviour change: A systematic review and metaanalysis of the impact of theoretical basis, use of behaviour change techniques, and mode of delivery on efficacy. Journal of Medical Internet Research, 12(1):e4, 2010. 